\title{
Anabases
}

ANABASES Traditions et réceptions de l'Antiquité

$4 \mid 2006$

Varia

\section{Recherches et projets de travail sur la Bibliothèque historique de Diodore de Sicile}

\section{Dino Ambaglio}

\section{(2) OpenEdition}

1 Journals

Édition électronique

URL : http://journals.openedition.org/anabases/3017

DOI : 10.4000/anabases.3017

ISSN : 2256-9421

Éditeur

E.R.A.S.M.E.

\section{Édition imprimée}

Date de publication : 1 octobre 2006

Pagination : 273-296

ISSN : 1774-4296

\section{Référence électronique}

Dino Ambaglio, "Recherches et projets de travail sur la Bibliothèque historique de Diodore de Sicile », Anabases [En ligne], 4 | 2006, mis en ligne le 01 janvier 2012, consulté le 21 octobre 2019. URL : http:// journals.openedition.org/anabases/3017 ; DOI : 10.4000/anabases.3017

Ce document a été généré automatiquement le 21 octobre 2019.

(c) Anabases 


\title{
Recherches et projets de travail sur la Bibliothèque historique de Diodore de Sicile
}

\author{
Dino Ambaglio
}

\section{NOTE DE L'AUTEUR}

Je remercie Jérémy Direz, jeune étudiant d'histoire antique, pour son aide dans la traduction française de ces lignes.

1 Y a-t-il encore un sens aujourd'hui à s'occuper sérieusement de Diodore et de son chantier historiographique, après des siècles de Quellenforschung qui l'ont littéralement déconsidéré et des générations d'éminents chercheurs qui ne l'ont pas épargné de leurs jugements féroces, sarcastiques et - ce qui est le pire - fondamentalement justes ? Y a$\mathrm{t}$-il encore un sens à discuter de son mode de travail et à chercher à en tirer quelque utilité, au-delà de l'utilisation pure et simple que nous faisons de la Bibliothèque, quand nous la citons dans nos travaux pour des faits de l'histoire antique qui, autrement, resteraient inconnus, ou pour quelque version différente concernant les événements et la chronologie? La publication, sous la direction de l'auteur de ces lignes, des Atti del Convegno "Epitomati ed epitomatori : il crocevia di Diodoro Siculo", Pavie, 21-22 avril 2004, in Syggraphé 7, Edition New Press, Côme, 2005, 247 p., voudrait donner une réponse affirmative à cette question. Cependant on se doit de dire d'emblée qu'on a écarté par hypothèse toute tentative pour revaloriser Diodore en confrontant les résultats aux propositions qu'il avance dans son préambule, parce qu'il faut toujours avoir à l'esprit que Diodore travaillait pour composer une «vue d'ensemble de l'histoire grecque et romaine ", ainsi que l'a observé E. Gabba.

2 Naturellement, il serait pour le moins inopportun que l'éditeur du volume des Actes du colloque fût seul à établir une recension ; à l'inverse, il peut-être utile, en profitant de la généreuse et amicale invitation de Pascal Payen d'intervenir dans Anabases à propos 
des initiatives touchant aux études diodoréennes, d'esquisser quelques réflexions concernant l'utilité toujours actuelle de la Bibliothèque. Le colloque pavesan permet aussi de faire le point sur les recherches consacrées à Diodore en Italie. Il faut en effet indiquer, comme témoignage de la vitalité, en Italie, des études sur l'historien sicilien, quelques mois avant que ne se tienne le colloque de Pavie, qu'une autre manifestation s'est déroulée à Milan, les 15-16 janvier 2004: Diodoro e l'altra Grecia. Macedonia, Occidente, Ellenismo nella Biblioteca storica, dont les Actes ont été publiés sous la direction de Cinzia Bearzot et Franca Landucci, Milan, Vita e Pensiero, 2005. Du reste, sur Diodore, depuis quelques années, travaillent en collaboration des groupes de recherche de l'Université de Bologne, de l'Université Catholique de Milan, des Universités de Florence et de Pavie ; ces projets ont été presque toujours accueillis et financés par des collectivités locales et par le Ministère de l'Université et de la Recherche Scientifique. Actuellement la publication des commentaires des livres XIII et XVIII est imminente, tandis que les autres volumes relatifs aux livres V, XII, XV, XXIII-XXIV sont dans un état d'élaboration déjà avancé, et les autres vont être mis en chantier.

Dans les exposés et les discussions du colloque pavesan, auquel ont apporté leur contribution E.Gabba, S. Bianchetti, A.Visconti, P.A.Tuci, R. Vattuone, C.Cuscunà, U. Bultrighini, C. Bearzot, M. Bertoli, G. Mafodda, E. Bianco, V.Costa, F. Landucci, N. Cusumano, G. Vanotti, C.Zizza, les points qui sont apparus essentiels pour les recherches présentes et futures sur la Bibliothèque historique peuvent être présentés ainsi.

4 L'œuvre de Diodore, avec ses prétentions universelles sur les plans chronologique et spatial, peut être définie comme un miroir dans lequel se reflète l'élargissement du monde produit par l'action militaire et politique de Rome. Il est assez logique, mais rarement rappelé, que n'importe quelle œuvre historiographique d'ambition universelle, ou d'une façon ou d'une autre tournée vers un temps révolu, est le produit, au moins à titre préliminaire, d'un travail de compilation de la tradition historiographique précédente. C'est dans ce sens que nous pouvons parler de Diodore comme d'un compilateur compilé, mais il en va de même pour beaucoup d'auteurs appartenant à l'historiographie grecque. Il suffit de penser au cas exemplaire d'Arrien et de son Anabase d'Alexandre et, par la suite, dans le cadre de la littérature qui fond et confond géographie et histoire, à Strabon avec sa Géographie qui se situe sur la même ligne de travail. Il s'agit dans ces cas d'une forme de résumé et de reconstruction "noble", c'est-à-dire enrichie par les choix et par les fréquentes interventions personnelles des auteurs, qui sont des professionnels de l'histoire et qui ne méritent pas d'être comparés aux tardifs compilateurs sans visage et sans nom chargés, par exemple, par l'empereur Constantin Porphyrogénète de construire des anthologies dans lesquelles l'intérêt pour les faits et les rapports de cause à conséquence se noient dans un moralisme quelque peu obsédant.

En tant qu'épitomé, la Bibliothèque constitue un précieux réservoir d'extraits historiographiques, grâce auxquels nous pouvons accroître notre connaissance des auteurs disparus et surtout mieux raisonner sur l'évolution de cette même historiographie antique. La Quellenforschung, parfois utilisée à outrance par les chercheurs modernes à propos de Diodore, comme étant seule digne d'intérêt, peut produire encore quelques fruits, surtout pour ce qui regarde certaines sources locales parvenues à Diodore non pas directement, mais à travers les auteurs qu'il cite 
habituellement; cette voie d'analyse confirme l'importance de la Bibliothèque pour la survie de filons différents de ceux de la tradition « vulgaire».

6 Pour ce qui concerne les questions historiques qui ont été débattues, la contribution personnelle de Diodore est positive et suffisamment claire lorsqu'il tente de montrer au lecteur en quoi les autres versions se contredisent; ce qui est surtout important est sa conscience de la distance entre discours et fait historique : cette attitude est tout autre que banale, bien que personne ne puisse dire si cette observation a été formulée à l'origine par Diodore.

7 Le lexique de la Bibliothèque, souvent jugé peu ou nullement intéressant, et en conséquence ignoré en raison de son imprécision et du caractère uniforme et mécanique de ses choix, toujours semblables dans des circonstances historiques et chronologiques très différentes entre elles, peut être encore utilement étudié.

8 La carte géographique de Diodore, imaginaire sous certains aspects et identifiée par hypothèse avec la carte dite d'Éphore, présente au second plan de l'œuvre historique et impliquée par le récit des faits, mérite d'être encore prise en considération, parce qu'elle comporte un plan des espaces qui est le fruit d'une longue élaboration culturelle.

9 L'entrecroisement entre histoire et biographie dans la Bibliothèque (et dans ses sources) anticipe sur des développements proches et fondamentaux de la biographie politicomilitaire: cet aspect encore inexploré aujourd'hui, comme le remarquait déjà L. Piccirilli, mérite d'être approfondi.

10 Si toutes ces pistes ne sont pas sans fondement, il reste encore à faire sur Diodore, comme toujours et par chance.

\section{AUTEUR}

DINO AMBAGLIO

Università degli Studi di Pavia

ambaglio@unipv.it 